\title{
RELACIÓN ENTRE EL SÍNDROME DE BURNOUT Y LA CALIDAD DE VIDA DEL PROFESIONAL DE ENFERMERÍA DE LA CLÍNICA GOOD HOPE, JULI0 2009
}

\author{
Marisol SOTELO ROSALES', Danitza CASILDO BEDÓN², Fany MAMANI CHECYA ${ }^{3}$
}

\begin{abstract}
RESUMEN
Objetivo: Determinar la relación que existe entre síndrome de Burnout y la calidad de vida de los enfermeros de la Clínica Good Hope. Metodología: Se realizó un estudio de corte transversal correlacional. El universo elegido estuvo constituido por 35 enfermeras de la Clínica Good Hope en el mes de julio del año 2009. Se utilizó el cuestionario de Maslach Burnout Inventory para medir el síndrome de Burnout y el SF-36 de Medical Outcomes Study para la calidad de vida. Los datos fueron procesados utilizando el paquete estadístico SPSS v.15 a través de los cuales se realizaron las pruebas de hipótesis para la Chi cuadrada con un nivel de confianza 95\%. Resultados: Como resultado se encontró que el promedio obtenido del total de encuestas según el cuestionario Maslach en el área de agotamiento emocional fue de 26 lo que indica un nivel medio, del mismo modo en el área de despersonalización se obtuvo un promedio de 10 el mismo que indica un nivel de despersonalización media; por otro lado, en el área de realización personal se obtuvo un promedio 49, el cual indica un nivel bajo. Con respecto a las 8 dimensiones de la Calidad de Vida, la Función Física presenta la puntuación máxima de 92, seguidamente el Rol Físico con 88 y en el Rol Emocional con 80; en las puntuaciones menores que corresponden a Deficiente Calidad de Vida son Salud General, Vitalidad con 66 de promedio y 70 en Dolor Corporal. El R de Pearson indica que la prueba del agotamiento emocional se relaciona significativamente con la función física $(\mathrm{P}=0.003<0.05)$, con el dolor corporal $(\mathrm{P}=0.048$ $<0.05)$, con la salud general $(0.013<0.05)$, con la vitalidad $(\mathrm{P}=0.003<0.05)$, con la función social $(\mathrm{P}=0.041<$ $0.05)$ y con la salud mental $(\mathrm{P}=0.002<0.05)$, así también se puede observar relación significativa en la realización personal con la función física $(\mathrm{P}=0.004<0.05)$, con la vitalidad $(\mathrm{P}=0.013<0.05)$ y con la salud mental $(\mathrm{P}=0.013$ $<0.05)$. Conclusión: Las enfermeras de la Clínica Good Hope tienen mejor calidad de vida en la función física, rol físico y rol emocional. Asimismo, las enfermeras presentan en promedio nivel medio de agotamiento emocional y despersonalización, así como nivel bajo de despersonalización. Cabe resaltar que las enfermeras se encuentran con tendencia a desarrollar este síndrome; por lo tanto, sugerimos llevar a cabo programas preventivos que se ajusten a la necesidad específica de la institución para mantener elevado grado de satisfacción de las enfermeras y pacientes.
\end{abstract}

Palabras Clave: Síndrome de Burnout, estrés laboral, desgaste profesional, calidad de vida.

\begin{abstract}
Objective: To determine the relationship that exists between the Burnout Syndrome and the Quality of Life in nurses from the "Good Hope" Clinic. Methodology: A transversal correlational study was performed. The selected universe was composed of 35 nurses from the "Good Hope" Clinic in July of 2009. The Maslach Burnout Inventory Questionnaire was used to measure burnout syndrome, and the SF-36 Medical Outcomes Study was used for the quality of life. The data were processed using the SPSS v.15 statistical package through which the tests of hypotheses were performed for the chi-square with 95\% confidence level. Results: As results, according to Maslach Questionnaire, which was obtained from all the surveys, it was found that the average in the area of emotional burnout was 26 indicating a medium level; in the same way in the area of depersonalization it was obtained an average of 10 which indicates a media depersonalization level. On the other hand in the area of personal accomplishment it was obtained an average of 49 which indicates a low level. With respect to the 8 dimensions of quality of life: physical function has a maximum score of 92, then with 88 for physical role, and 80 for emotional role. The lower scores correspond to poor quality of life, these are: general health, vitality average of 66 and 70 for bodily pain. The Pearson R test indicated that emotional burnout was significantly related to physical function $(\mathrm{P}=0.003<0.05)$, bodily pain $(\mathrm{P}=$
\end{abstract}

\footnotetext{
${ }^{1}$ Licenciada en Enfermería, Especialista en Emergencias y Desastres, Docente de la Universidad Peruana Unión

${ }^{2}$ Licenciada en Enfermería, Especialista en Emergencias y Desastres, Enfermera de la Clínica San Felipe

${ }^{3}$ Licenciada en Enfermería, Especialista en Emergencias y Desastres, Enfermera de la Clínica Good Hope
} 
$0.048<0.05)$, general health $(0.013<0.05)$, vitality $(\mathrm{P}=0.003<0.05)$, social function $(\mathrm{P}=0.041<0.05)$ and mental health $(\mathrm{P}=0.002<0.05)$, on the other hand we can observe significant relationship in the personal accomplishment with physical function $(\mathrm{P}=0.004<0.05)$, vitality $(\mathrm{P}=0.013<0.05)$ and mental health $(\mathrm{P}=0.013<0.05)$. Conclusions: Nurses from the "Good Hope" Clinic have better quality of life in physical function, physical role, and emotional role. These nurses also have an average mean level of emotional burnout and depersonalization and low levels of depersonalization. It should be noted that nurses are in tendency to develop this syndrome; therefore we suggest carrying out preventive programs that meet the specific needs of the institution to maintain a high degree of satisfaction of nurses and patients.

Key words: Burnout Syndrome, Work Stress, Career Burnout, Quality of Life.

\section{INTRODUCCIÓN}

El Síndrome de Burnout fue introducido y conocido por primera vez en la década de los 70 por Freudenberger (1974), quien lo definió como un estado de fatiga o frustración que se produce por dedicación a una causa, forma de vida o de relación.

Por otro lado, Gil-Monte \& Peiró (1997), definen al síndrome de Burnout como una respuesta al estrés laboral crónico, integrado por actitudes y sentimientos negativos hacia las personas con las que se trabaja: actitudes de despersonalización, falta de realización profesional en el trabajo y también por la vivencia de encontrarse emocionalmente agotado.

En la actualidad se acepta que el Síndrome de Burnout afecta a cualquier grupo ocupacional. Existen profesionales más predispuestos a este síndrome, como es el caso de los profesionales de la salud, que experimentan una variedad de problemas de salud en el aspecto físico que se manifiesta con cefaleas, úlceras pépticas y trastornos cardiovasculares. Por otro lado, en el aspecto psicológico se evidencian problemas como estrés, depresión y ansiedad.

Asimismo, García (2001) refiere que la calidad de vida del profesional de enfermería es el sentimiento de bienestar que se deriva del equilibrio entre las demandas o cargas de la profesión y los recursos psicológicos, organizacionales y relacionales del individuo.

No debe dudarse el hecho de que las personas tienden a dar calidad al cliente en el mismo grado que perciben que son tratadas por la organización en donde trabajan. Por ello es importante que la "Calidad de Vida Profesional” sea medida periódicamente y sea objeto de un proceso de mejora continua en el seno de las organizaciones.

En este estudio las autoras consideran que la enfermera es vulnerable a padecer síndrome de Burnout, pues está sometida a altos niveles de estrés y tensión por la responsabilidad que asume en sus horas laborales, la sobrecarga de trabajo, el continuo contacto con pacientes críticos, con enfermedades muchas veces terminales, contagiosas y el contacto con el dolor y la muerte; además de los escasos períodos de descanso, la falta de cooperación de los pacientes por su estado de gravedad y el aumento de las exigencias en cuanto a la calidad de atención del profesional de enfermería.

En el servicio de emergencia, hospitalización, SOP y UCI de la Clínica Good Hope se observó algunas enfermeras cansadas, sin ganas de seguir trabajando; refieren cefalea, contracturas musculares, ansiedad por regresar a sus hogares. Otras enfermeras se encontraban muy agotadas refiriendo realizar turnos extras por falta de personal, y demanda de pacientes.

En respuesta a esta problemática se realizó el presente trabajo de investigación, teniendo como objetivo principal determinar la relación que existe entre síndrome de Burnout y la calidad de vida de los enfermeros de la Clínica Good Hope. Siendo los objetivos específicos: Determinar los niveles del Síndrome de Burnout y determinar la calidad de vida de los enfermeros de la Clínica Good Hope julio 2009

\section{MATERIAL Y MÉTODOS}

Este estudio fue realizado en la Clínica Good Hope en julio del año 2009 y se encuestaron a 35 profesionales de enfermería. Los criterios de inclusión fueron: enfermeras de profesión que trabajan más de un año en la institución, que están en actividad, independientemente de la forma de contrato; y los criterios de exclusión fueron: enfermeras con licencias por maternidad, descanso médico y vacaciones.

El diseño del estudio fue cuantitativo, descriptivo, correlacional y de corte transversal. Se aplicó como instrumento de recolección de datos el cuestionario de Maslach Burnout Inventory para medir el síndrome de 
Burnout, el mismo que cuenta con 22 ítemes, los cuales evalúan las dimensiones de agotamiento o cansancio emocional (9 ítemes), despersonalización (5 ítemes) y la falta de realización personal (8 ítemes).

Para medir la Calidad de Vida se empleó el cuestionario SF-36 de Medical Outcomes Study que evalúa la función física (10 itemes), función social (2 ítemes), limitaciones del rol de problemas físicos (4 ítemes), limitaciones de problemas emocionales (3 ítemes), salud mental (5 ítemes), vitalidad (4 ítemes), dolor corporal (2 ítemes ) y percepción general de la salud (6 ítemes).

Para el análisis estadístico se utilizó el software SPSS v.15; los datos obtenidos fueron vaciados a la matriz de datos del software estadístico para su respectivo análisis; se empleó la prueba de r de Pearson para determinar el nivel de significancia

\section{RESULTADOS}

A continuación detallamos los resultados obtenidos:

En la tabla $\mathrm{N}^{\circ} 1$ se observa que el $57.1 \%$ de las enfermeras son solteros y el $68.6 \%$ de las edades oscila entre 20 a 30 años de edad.

Tabla 1. Puntuaciones promedio del Síndrome de Burnout en el profesional de enfermería de la Clínica Good Hope, julio 2009

\begin{tabular}{|ccc|}
\hline \multicolumn{1}{|c}{ DIMENSIONES } & $\begin{array}{c}\text { PROMEDIO } \\
(\mathbf{X})\end{array}$ & $\begin{array}{c}\text { NIVELES DE } \\
\text { IDENTIFICACIÓN }\end{array}$ \\
\hline Agotamiento Emocional & 26 & Medio \\
Despersonalización & 10 & Medio \\
Realización personal & 49 & Bajo \\
\hline Fuente: Instrumento aplicado a los enfermeros de la CGH, 2009
\end{tabular}

La tabla 1 muestra el promedio de puntuación obtenido del total de encuestas según el cuestionario Maslach, en el área de Agotamiento Emocional fue de 26, lo que indica que las enfermeras padecen un nivel medio, asimismo, en el área de Despersonalización se encuentran un nivel medio con puntuación de 10. Por otro lado, en la Realización Personal se obtuvo una puntuación de 49 ubicándose en un nivel bajo.

Tabla 2. Puntuación promedio de la calidad de vida en el profesional de enfermería de la Clínica Good Hope, julio 2009

\begin{tabular}{lc|}
\hline DIMENSIONES & PROMEDIO (X) \\
\hline Función Física & 92 \\
Rol Físico & 88 \\
Dolor Corporal & 70 \\
Salud General & 66 \\
Vitalidad & 66 \\
Función Social & 75 \\
Rol Emocional & 80 \\
Salud Mental & 74 \\
\hline
\end{tabular}

Fuente: Instrumento aplicado a los enfermeros de la CGH, 2009 
Esta tabla 2 muestra que de las 8 dimensiones de la Calidad de Vida, la Función Física presenta la puntuación máxima de 92, seguidamente el Rol Físico con 88 y el Rol Emocional con 80. Por otro lado, las puntuaciones menores que indican deficiente Calidad de Vida son Salud General, Vitalidad con 66 y Dolor Corporal con 70.

Tabla 3. Relación entre Calidad de Vida y el Síndrome de Burnout en el profesional de enfermería de la Clínica Good Hope, julio 2009

\begin{tabular}{lcccccccc}
\multicolumn{1}{c}{$\begin{array}{c}\text { Dimensión de calidad } \\
\text { de vida }\end{array}$} & $\begin{array}{c}\text { Función } \\
\text { física }\end{array}$ & $\begin{array}{c}\text { Rol } \\
\text { físico }\end{array}$ & $\begin{array}{c}\text { Dolor } \\
\text { Corporal }\end{array}$ & $\begin{array}{c}\text { Salud } \\
\text { General }\end{array}$ & Vitalidad & $\begin{array}{c}\text { Función } \\
\text { Social }\end{array}$ & $\begin{array}{c}\text { Rol } \\
\text { emocional }\end{array}$ & $\begin{array}{c}\text { Salud } \\
\text { Mental }\end{array}$ \\
\hline Dimensión de Burnouta & 0.003 & 0.49 & 0.048 & 0.013 & 0.003 & 0.041 & 0.400 & 0.002 \\
$\begin{array}{l}\text { Agotamiento } \\
\text { Emocional }\end{array}$ & 0.14 & 0.92 & 0.17 & 0.69 & 0.63 & 0.40 & 0.21 & 0.69 \\
$\begin{array}{l}\text { Despersonalización } \\
\text { Realización Personal }\end{array}$ & 0.04 & 0.86 & 0.62 & 0.12 & 0.013 & 0.25 & 0.50 & 0.013
\end{tabular}

Fuente: Instrumento aplicado a los enfermeros de la CGH, 2009

El análisis de correlación estadística que se muestra en la tabla número 3 revela que el agotamiento emocional se relaciona significativamente con la función física $(\mathrm{P}=0.003<0.05)$, con el dolor corporal $(\mathrm{P}=0.048<0.05)$, con la salud general $(0.013<0.05)$, con la vitalidad $(\mathrm{P}=0.003<0.05)$, con la función social $(\mathrm{P}=0.041<0.05)$ y con la salud mental $(\mathrm{P}=0.002<0.05)$, por otro lado podemos observar relación significativa en la realización personal con la función física $(\mathrm{P}=0.004<0.05)$, con la vitalidad $(\mathrm{P}=0.013<0.05)$ y con la salud mental $(\mathrm{P}=0.013<0.05)$.

\section{DISCUSIÓN}

En la investigación realizada en la Clínica Good Hope se llegó a la conclusión de que existe presencia de Síndrome de Burnout, a nivel medio en la dimensión de agotamiento emocional y en la dimensión de despersonalización. Se encontró; asimismo, un nivel bajo en la dimensión de realización personal.

Los resultados del presente estudio son congruentes con los hallazgos de Quiroz y Saco (1999) en la investigación "Factores asociados al Síndrome de Burnout en médicos y enfermeras del Hospital Nacional Sur Este de EsSalud del Cusco”, mediante el cual se determinó que el Síndrome de Burnout bajo se presenta en el $79.7 \%$ de los médicos y $89 \%$ de las enfermeras; en grado medio en el $10.9 \%$ de médicos y $1.4 \%$ de enfermeras, asociados a la jornada laboral mayor de 40 horas semanales, menos de 6 horas de esparcimiento semanal y otros trabajos fuera del hospital.

Con respecto a la calidad de vida en la dimensión de salud mental, el $10 \%$ de las enfermeras presentan un nivel casi óptimo (92-96 puntos), y en la dimensión de vitalidad el $9 \%$ de las enfermeras tienen un nivel casi óptimo (90-95 puntos). Sólo el $15 \%$ de las enfermeras alcanzaron un nivel óptimo en relación con la percepción de la salud. 


\section{REFERENCIAS BIBLIOGRÁFICAS}

1. Fundación Prenent. Jornada sobre Burnout. En Gaceta Médica 1 (8 de junio; 2003). Barcelona; p. 6.

2. Martos Medina, J. A.. Guía de la Prevención del estrés. Cuídate. SATSE. Madrid; 1999.

3. Ramón Sánchez Manzanera. Cuidados Paliativos. Avances sin final. Edit.

4. Asociación para la Formación Continuada en Ciencias de la Salud y Educación.Alcalá. Madrid. 2003; pp. 494-495.

5. De las Cuevas Castresana, C. El desgaste profesional en atención primaria: Presencia y distribución del Síndrome de Burnout en personal sanitario. Anales de Psiquiatría, no 10. 1994. pp. 180-184.

6. Ramón Sánchez Manzanera. Resumen de la clínica del Síndrome de Burnout en cuidados paliativos:
Avances sin final. Edit. Asociación para la Formación continuada en Ciencias de la Salud y Educación. Alcalá. Madrid; 2003. p. 498.

7. López Soler, M.T.: Síndrome de Burnout en cuidados paliativos: Avances sin final. Edit. Alcalá. Madrid; 2003. pp. 498-503.

8. Flórez Lozano, J.A.. Síndrome de "estar quemado”. Edit. Edika Médica. España; 2003; 67.

9. Mingote Adán, J.C. Pérez García, S.: Estrés en la Enfermería. El cuidado del cuidador. Edit. Díaz de Santos. Madrid; 2002. pp. 92-95.

10. Organización Panamericana de la Salud. Perfil del Sistema de Servicios de Salud del Perú, Programa de organización y Gestión de sistemas y Servicios de salud, División de Desarrollo de Sistemas y Servicios de Salud; 2001. pp. 1-3. 